\section{La Cantuta y la educación nacional}

En el tiempo trascurrido desde que empecé a caminar por los senderos de la sin gular topografía cantuteña, no ha desmayado en mi esta mistica docente que no es otra cosa que el espíritu y la imagen siempre presentes del Dr. Peñaloza y de los maestros que junto a él se abrieron paso con energía, pero con prudencia; con sencillez, pero con seguridad; muy sabiamente, pero con modestia.

La evocación del ambiente de la Escuela $y$ el eco de las voces cariñosas de los maes. tros que nunca olvidaré, confirman y reafirman ese espiritu, esa fuerza docente que nos seguirá impulsando, que duda cabe, para seguir sembrando y preparando nuevos almácigos y continuar la nobilísima tarea de "educar al hombre en todo lo que tiene de hombre".

$Y$ que brillante ocasión la que nos brinda hoy la vida, de asistir a la presentación del testimonio más importante de un maestro que supo enfrentar la realidad del sistema entonces imperante para empezar su tarea que aún no termina.

Que importante es esta convocatoria porque considero que se estaría iniciando la segunda etapa de este gran proyecto que finalmente logrará cambiar la faz de nuestra patria $\vee$ de la América hispana. Debemos considerar que sólo se ha hecho una pausa para tomar impulso y vigorizar e imponer la doctrina pedagógica que los pueblos esperan.

He leido con particular entusiasmo las páginas del libro que hoy se comenta $y$ he tenido la nitida impresión de asistir a una de las innumerables clases magistrales del $\mathrm{Dr}$. Waiter quien con gran sencillez y claridad desarrolla lo que me permite calificar como la "doctrina Peñaloza", que de manera singular ta hemos vivido muchos peruanos $y$ que sigue aún motivando nuestro quehacer docente.

Permitame por esta única vez, expresar aspectos contenidos en este valioso documento, a través de las vivencias que tuve en el entorno $y$ dentro del singular ambiente cantuteño. Son once años a lo largo de los cuales vivi las experiencias más gratas de mi vida, algunas de la cuales mencionaré a continuación. Debo decir además que aparte de mi testimonio personal tengo la versión de siete hermanos míos los cuales son ex alumno de los planteles de Aplicación.

Pertenezco a la Promoción $1958 \mathrm{del}$ Co. legio de Aplicación de la Escuela Normal Superior. El nombre que lleva nuestra promoción es Walter Peñaloza y no es vanidad ni presunción el que yo diga en este ambien. te, que conozco la vida de la Escuela mejor que muchos, es más estoy segura de decirles que he observado a esta hermosa comunidad estudiantil desde sus inicios hasta su etapa de mayor auge.

La primera vez que escuché hablar de La Cantuta fue como referencia al lugar: $\mathrm{mi}$ familia, mi madre en especial nos narraban que cuando niños iban a la quebrada de "corte chico" y "corte grande" a pasear $y$ 
recolectar guayabas. Posteriormente, ya instalados en Chosica fue mi padre el que nos dio la noticia de la edificación de una Escue. la formadora de maestros que sería la continuación del Primer Instituto Pedagógico Nacional de Varones y tendría planteles de Aplicación. Entusiasmado como estaba nos inscribió y matriculó a cinco de nosotros. A partir de esos hechos mi familia, como muchas familias chosicanas, iniciaron sus rela. ciones con el Dr. Peñaloza y la nueva fami. lia cantuteña.

En un primer momento sentimos un poco de inquietud. Nos trasladaban a un nuevo colegio y era mixto, una gran novedad para el momento. Ingresé al 5 to de primaria, casi a mitad de año; fueron difíciles los primeros dias: nuevos compañeros, un ambiente distinto. Nuestro profesor era don Aquiles $\mathrm{Ni}$ cho Rosadio; él con su experiencia y gran capacidad para motivarnos nos fue introduciendo dentro de una pedagogía nueva; de un sistema vertical pasábamos a uno horizontal; de un programa rígido pasábamos a una programación que haciamos diariamente a las 8:15 a.m. y que comenzaba con ¿qué quieren hacer hoy día? Todos los pedidos eran registrados, hasta los que podían parecernos fuera de lugar, como pasearnos - jugar. Con gran habilidad conducía nues. tros pedidos. Finalmente teniamos un temario que desarrollábamos gustosamente, pues sentiamos que habíamos participado en la selección de los temas y nuestras opiniones no sólo habian sido escuchadas sino también tomadas en cuenta.

Realizábamos tareas que nos permitian desarrollar el aprendizaje de las matemáticas, del lenguaje, de la historia, la geografía, etc., junto a ellas desarrollábamos otras acti- vidades, como vender en la tienda escolar, sembrar en el huerto, volar cometas, dibujar, participar en representaciones teatrales y en todos los juegos planificados. En poco tiempo comprobamos un cambio: la amistad entre los compañeros de clase y actitudes solidarias y comprensivas dentro de un marco de sinceridad y respeto.

Los días lunes, reunidos en el ingreso principal, interveniamos en la presentación de un hecho trascendente, de una efeméride y escuclıábamos las orientaciones que nos brindaba el venerable Director don César Oré y Luque y los docentes de las otras aulas como Rosa Cervantes, Irene Piedra, Berta Rodríguez, Guillermo Paredes Calmet, Luis Torrejón, todos juntos con la mística y la ponderación de don Walter.

Todavía tengo muy presente el día que tuvimos que dar examen de madurez mental para entrar a la secundaria, parecía un examen de admisión a la universidad. Se dieron treinta vacantes y se presentaron doscientos postulantes. Naturalmente sólo entraron los que por puntaje $y$ estricto orden ocuparon los puestos correspondientes. Hubo presio. nes de todo tipo, pero, desde esos primeros momentos don Walter nos enseñaba con hechos el significado de la justicia y el respeto por la dignidad de las personas.

En el Colegio de Aplicación (el plantel secundario) nuestras experiencias fueron di. ferentes; era otra la organización pero percibimos la insistencia en formarnos en el campo del conocimiento, en el de las actividades $y$ en el campo del trabajo. Así lo fuimos comprobando con don Manuel Moreno en las clases de Castellano, con don Walter Peñaloza en Historia Universal; en Biología con don Pedro Coronado; en Historia Nol 
Perú con don Roberto Rioja; en Platemáticas con Tito Viacava; en Geografía con e! profesor Peñaherrera; en Dibujo con dan Miguel Reynel. Además haciamos labores de campo con dón Gamaniel Pérez, trabajos de carpintería con el profesor Musayón; hacia. mos teatro con el profesor Galli y Educa. ción Física con nuestros queridos profesores Hilda Zelaya y Sebastián Guillén. Nos sepa. rábamos los hombres de las mujeres para que ellos recibieran Instrucción Premilitar y nosotras Economía Doméstica con doña Hilda de Moreno. Practicamos la esgrima, el ballet, la natación, el atletismo y otros juegos deportivos. Con el trascurrir de los años fuimos cambiando de profesores y recibimos clases de hombres singulares por su capacidad y trayectoria tales como don. Pedro Benvenuto, don Luis Jaime Cisneros, don Guillermo Daly, don Luis Alberto Ratto, Celia Albrizio, el Dr. Vega, don César Pacheco Vélez, monseñor Lituma, el padre de la Calzada, don César Carranza, el profesor Velásquez, el profesor Huaringa, don Godofredo Bendezú, doña Teodora R. de Rocha, el profesor Cenzano, doña Clotilde Albarracín, don Pedro Cueva, Gerardo Ayzanoa y otros profesores más.

Sabíamos que nuestras aulas eran laboratorios donde se ensayaban programas y métodos; donde los futuros docentes realizaban sus primeras experiencias; las prácticas de los profesores alumnos algunos conocedores del tema presentado, ecuánimes con una personalidad docente ya definida, otros inseguros, faltos de preparación mejoraban cada vez más en sucesivas clases.

No teníamos auxiliares, había profesores consejeros, atentos a nuestra actuación $y$ a nuestro desarrollo académico y personal; se nos insoiró y preparó en la práctica del autocontrol y la autocrítica. Frente a nuestras inquietudes juveniles, el juego, el compañerismo, la solidaridad, la participación, ellos nos orientaron permanentemente.

En las aulas cantuteñas asistimos a la configuración del perfil docente $y$ ese arquetipo de maestro es el que logramos internalizar a través de nuestras experiencias nersonales en cada etapa de nuestra formación magisterial. Cuando muchos de nosotros participamos de la Reforma Eúucativa del año 1968 comprobamos que ya la habiamos experimentado $y$ vivido con excelentes resultados.

Sería vanidoso de mi parte si me atre viera a presentar conclusiones en torno a este hecho tan importante. No puedo dejar de señalar sin embargo la trascendencia de este importante acontecimiento a través de los siguientes hitos:

1. La Joctrina Eúucativa Peñaloza-Cantuta abre una importante y decisiva inquietud que seriala una orientación moderna, que rompe los modelos tradicionales de instrucción.

2. Se inicia una forma distinta de instrucción y formación de docentes que imbuidos de una mistica educativa bien cimen. tada han difundido en el ámbito nacional y continental los valores esenciales aprendidas en La Cantuta.

3. La Reforma Educativa planteada en la época militar considera y retoma aspectos importantes de lo que podemos llamar Dectrina Peñaloza. Ahora percibo con claridad que el diseño de Reforma Educativa se inspiró esencialmente en los logros concebidos en ese importante la. boratorio que fue la Escuela Normal de 
La Cantuta.

Concluidos los estudios en el Colegio de Aplicación segui estudios profesionales en la Escuela Normal Superior y de ellos guardo experiencias inolvidables.

Desde mi perspectiva de Docente en la Universidad Nacional Agraria, puedo afirmar que la vida académica de esta Universidad fue enriquecida por los aportes que nuestro querido Walter y varios de sus colaboradores introdujeron en ella.

Finalmente quiero expresar mi reconocimiento imperecedero a don Víalter, a su obra pedagógica de invalorable trascendencia $y$ al testimonio bibliográfico que hoy nos convoca. Mi aprecio y mi homenaje a los docentes que aún nos acompañan y mi recuerdo y homenaje para aquellos profesores que estarán siempre en nuestra memoria.

Flora Benavides de Abanto

\section{Congreso de sociología latinoamericana}

Del 28 al 31 de mayo de 1991 se realizó en el Palacio de Convenciones de La Habana, Cuba, el XVIII Congreso de la Asociación Latinoamericana de Sociología (A. LAS). El Congreso se realizó con alrededor de mil delegados no sólo sociólogos, antropólogos, sicólogos, abogados, trabajadores sociales, etc., tanto estudiantes como profesionales.

La Sociología Latinoamericana se debate actualmente en un proceso profundo de trasformación que tiende a dejar atrás a los viejos paradigmas de análisis como fueron el rechazo ciego al capitalismo y a la modernización por un socialismo que se consideraba perfecto y humanizante; como el exceso de confianza en el impacto de las estructuras sabre los hombres, quitándoles su propia subjetividad y acción creadora independientemente de las mismas.

Asimismo esta sociología de los setenta tenía un aprecio y esperanza ingenua en la confrontación implacable entre las fuerzas renovadoras y retardatarias sobre el consenso y la negociación; por los intereses de grupo sobre los individuales; por el iluminismo de las minorías que "representaban" a las grandes mayorías, dejando de lado la riqueza de su vida cotidiana y la racionali- 